Applied Remote Sensing

\title{
Polarimetric synthetic aperture radar image unsupervised classification method based on artificial immune system
}

\author{
Yu Jie \\ Wang Gang \\ Zhu Teng \\ Li Xiaojuan \\ Yan Qin
}




\title{
Polarimetric synthetic aperture radar image unsupervised classification method based on artificial immune system
}

\author{
Yu Jie, ${ }^{\text {a,b,c,* }}$ Wang Gang, ${ }^{\mathrm{d}}$ Zhu Teng, ${ }^{\mathrm{c}}$ Li Xiaojuan, ${ }^{\mathrm{b}, \mathrm{e}}$ and Yan Qin ${ }^{\mathrm{c}}$ \\ ${ }^{a}$ Capital Normal University, State Key Laboratory Incubation Base of Urban Environmental \\ Processes and Digital Simulation, Beijing 100048, China \\ ${ }^{\mathrm{b}}$ Capital Normal University, College of Resource Environment and Tourism, Beijing 100048, \\ China \\ ${ }^{\mathrm{c}}$ Wuhan University, School of Remote Sensing and Information Engineering, 129 Luoyu Road, \\ Wuhan 430079, China \\ ${ }^{\mathrm{d}}$ Institute of Surveying and Mapping, Qingdao 266031, China \\ ${ }^{\mathrm{e}}$ Capital Normal University, Beijing Key Laboratory of Resource Environment and Geographic \\ Information System, Beijing 100048, China
}

\begin{abstract}
An unsupervised classification method based on the $H / \alpha$ classifier and artificial immune system (AIS) is proposed to overcome the inefficiencies that arise when traditional classification methods deal with polarimetric synthetic aperture radar (PolSAR) data having large numbers of overlapping pixels and excess polarimetric information. The method is composed of two steps. First, Cloude-Pottier decomposition is used to obtain the entropy $H$ and the scattering angle $\alpha$. The classification result based on the $H / \alpha$ plane is used to initialize the AIS algorithm. Second, to obtain accurate results, the AIS clonal selection algorithm is used to perform an iterative calculation. As a self-organizing, self-recognizing, and self-optimizing algorithm, the AIS is able to obtain a global optimal solution and better classification results by making use of both the scattering mechanism of ground features and polarimetric scattering characteristics. The effectiveness and feasibility of this method are demonstrated by experiments using a NASA-JPL PolSAR image and a high-resolution PolSAR image of Lingshui autonomous county in Hainan Province. () The Authors. Published by SPIE under a Creative Commons Attribution 3.0 Unported License. Distribution or reproduction of this work in whole or in part requires full attribution of the original publication, including its DOI. [DOI: 10.1117/1.JRS.8.083679]
\end{abstract}

Keywords: artificial immune system; polarimetric synthetic aperture radar image classification; immune clonal algorithm.

Paper 13510 received Dec. 11, 2013; revised manuscript received Jan. 7, 2014; accepted for publication Jan. 13, 2014; published online Feb. 13, 2014.

\section{Introduction}

Classification of ground-cover types is an important application of polarimetric synthetic aperture radar (PolSAR). ${ }^{1}$ One difficulty in PolSAR image classification is the large number of highly overlapping pixels and the large amount of excess polarimetric information, which makes classification very challenging. ${ }^{2}$ Over two decades, many approaches have been proposed to classify PolSAR images. In 1989, VanZyl suggested that PolSAR data could be classified into four scattering mechanisms, a discovery that had a significant impact on subsequent classifications. Cloude and Pottier ${ }^{3}$ later proposed an unsupervised classification algorithm based on another target decomposition theory. Eigenanalysis of the coherency matrix provides the polarimetric scattering mechanisms with matrix-characterizing parameters, such as the polarimetric entropy $H$ and the average polarimetric scattering angle $\alpha$. Using the $H / \alpha$ plane, all kinds of scattering mechanisms can be classified into eight basic zones to discriminate among surface

*Address all correspondence to: Yu Jie, E-mail: yuj2011@whu.edu.cn 
scattering, volume diffusion, and double-bounce scattering. Besides these scattering-mechanism studies, ${ }^{4,5}$ several classification approaches based on mathematical tools have been proposed, such as Markov random field models, ${ }^{6}$ region-growing techniques, ${ }^{7}$ and neural-network techniques. ${ }^{8}$ However, these techniques have yielded inconsistent results because classification performance has varied depending on which particular normalization scheme was selected.

In this study, a novel unsupervised classification model for PolSAR images based on the artificial immune system (AIS) has been developed in an effort to find a stable classifier. The AIS is a new method in the neural computation and evolutionary computation research field. ${ }^{9}$ It is designed to solve complex problems by exploring the biological immune system, obtaining its processing mechanisms, and developing appropriate engineering models. ${ }^{10}$ Unlike other evolutionary computation algorithms, the characteristics of the AIS, such as biological diversity, memory, tolerance, distributed parallel processing, and robustness, ensure a balance between exploration and exploitation. ${ }^{11}$ In recent studies, AISs have been applied to computer security, pattern recognition, machine learning, data mining, and function optimization. ${ }^{12}$ These studies have the common limitation of relying on training samples, which are treated as antibodies.

One point of innovation in this study is the introduction of the AIS into the clustering problem by actualizing the antigens and antibodies without any training sample. On the other hand, to combine the AIS with the PolSAR unsupervised classification algorithm in a reasonable way, an improved initialization method based on the genetic algorithm (GA) and a novel mutation operation has been proposed. Experimental results showed that this novel method can obtain a quasioptimal solution quickly and is effective for PolSAR image classification.

The rest of the paper is organized as follows. Necessary background information and fundamental knowledge are provided in Sec. 2. Details of the proposed unsupervised classification algorithm are described in Sec. 3. Section 4 describes the remote-sensing datasets used, together with experimental results and discussion. The conclusions are presented in Sec. 5.

\section{Background}

\subsection{Artificial Immune Systems}

AIS, inspired by the human immune system, has the powerful information-processing capabilities of the immune system. The concept originated from observations of how the defense mechanism of natural immune systems protects against attacks by antigens. Molecules such as viruses, bacteria, and fungi that can be recognized by the AIS are known as antigens. When a foreign antigen enters the body, the immune system can distinguish "self" from "nonself" and provide an immune response to exclude the antigenicity of nonself substances. ${ }^{13}$

The AIS algorithm is derived by abstracting the mechanism of the biological immune system. Many concepts and operators of the AIS algorithm correspond to the concepts and mechanisms of the biological immune system. The AIS is a product of the combination of biological immunology and computer science. ${ }^{14}$ In 1957, clonal selection theory was proposed by Burnet and is described below.

First, initialize the population, select the antigen and antibody randomly, calculate the affinity of all antibodies, and determine whether the termination criterion has been met; if it has, output the results, otherwise go to the next step.

Second, select the $n$-group antibodies that have the highest affinity with the antigen to generate a new set of antibodies and then perform the cloning operation on these selected antibodies. The result of this operation is a clonal selection set.

Third, perform the mutation operation on the clone collection; individuals with good antigen binding experience and poor variation opportunity. Then calculate the affinity of each antigen and each antibody and reselect those antibodies that have the highest affinity with each antigen.

Finally, replace the original antibodies with the lowest affinity with the new antibodies obtained from the reselection process so as to increase antibody diversity.

The clonal selection algorithm (CSA) is applied in the AIS, which is based on clonal selection and affinity maturation principles. ${ }^{15}$ Every generation in this algorithm includes the 
initiation of a candidate solution and selection, cloning, mutation, and reselection operations, similar to the GA. The AIS based on the CSA can deal with a complex search space and has the advantages of inherent parallelism and the ability to avoid local minima. ${ }^{16}$

\subsection{PoISAR Image Classifier}

\subsection{1 $H / \alpha$ decomposition}

Polarization is used to describe the wave characteristics of an observation point that changes over time; it is a common feature of various vector waves. ${ }^{17}$ According to the principles of fully polarimetric SAR measurement, the fully polarimetric SAR data make up the scattering matrix $\mathrm{S}$.

$$
S=\left[\begin{array}{ll}
S_{\mathrm{HH}} & S_{\mathrm{HV}} \\
S_{\mathrm{VH}} & S_{\mathrm{VV}}
\end{array}\right]
$$

where $\mathrm{S}_{\mathrm{HV}}$ is the scattering volume received horizontally and transmitted vertically. After decomposing the matrix $S$ based on the Pauli basis, the following vector can be obtained: ${ }^{3}$

$$
k=\frac{1}{\sqrt{2}}\left[S_{\mathrm{HH}}+S_{\mathrm{VV}} S_{\mathrm{HH}}-S_{\mathrm{VV}} 2 S_{\mathrm{HV}}\right]^{T},
$$

where $T$ indicates a transposed matrix. Then the coherency matrix $T_{3}$ can be written as

$$
T_{3}=k \times k^{* T},
$$

where * means the conjugate operation. Cloude et al. ${ }^{18}$ defined the polarimetric entropy $H$ and the scattering angle $\alpha$ of surface features according to their characteristics and their scattering mechanism by decomposing the coherency matrix. $H$ and $\alpha$ can be defined as follows:

$$
\begin{gathered}
H=\sum_{i=1}^{3}-p_{i} \log p_{i} \quad \text { where } p_{i}=\frac{\lambda_{i}}{\sum_{j=1} \lambda_{j}} \\
\alpha=\sum_{i=1}^{3} \alpha_{i} p_{i} .
\end{gathered}
$$

In Eq. (4), $\lambda_{1}, \lambda_{2}, \lambda_{3}$ are the eigenvalues of the coherent matrix $T_{3}$. The basic idea of $H / \alpha$ classification takes into account the average scattering mechanism and the scattering process and provides a quantitative description using the average angle and the scattering entropy $H$. Depending on the $H / \alpha$ plane, eight basic regions can be defined representing different scattering mechanisms, as shown in Fig. $1 .^{1}$

The scattering entropy $H$ is a measure of the randomness of scattering mechanisms. When $H=0$, this suggests that the surface features have only one major scattering mechanism and that the dominant scattering matrix is the eigenvector corresponding to the largest eigenvalue. When $H=1$, this indicates that the scattering targets are completely random and that their polarimetric information is 0 . The value of the scattering angle $\alpha$, which characterizes the physical scattering mechanism, represents, to a certain extent, the average scattering mechanism of the surface features and corresponds to a scattering process, which varies from surface scattering $(\alpha=0)$ to body scattering $(\alpha=45)$ to even scattering $(\alpha=90) .{ }^{17}$

\subsubsection{Wishart distance measurement}

Measuring the distance between a pixel and a cluster center is indispensable in a clustering problem. As clustering objects, pixels of the PolSAR image are expressed by the coherency matrix $T_{3}$ in this study. In 1999 , Lee et al. ${ }^{19}$ proposed the Wishart distance based on the 
Jie et al.: Polarimetric synthetic aperture radar image unsupervised classification method...

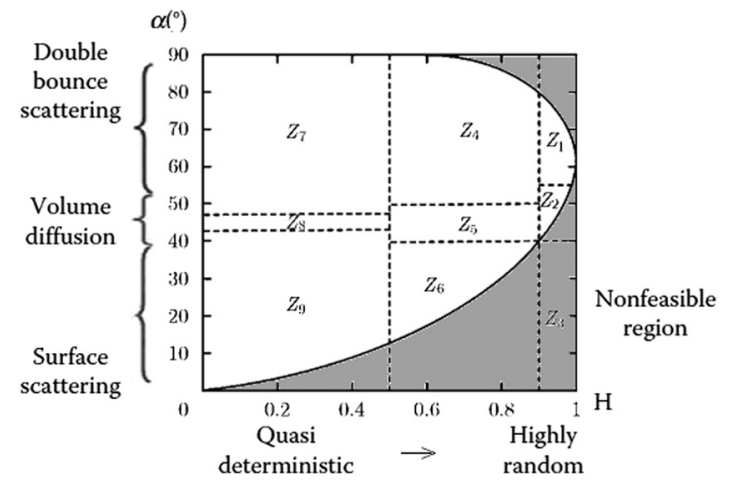

Fig. $1 \mathrm{H} / \alpha$ plane and its corresponding scattering mechanisms.

scattering mechanism. The distance between the coherent matrix $\langle\mathrm{X}\rangle$ of a pixel and the coherent matrix of the $m$ 'th class $m$ can be expressed as Eq. (6).

$$
d\left(\langle X\rangle, V_{m}\right)=\ln V_{m}+\operatorname{Tr}\left(V^{-1}\langle X\rangle\right),
$$

where $V_{m}$ is the cluster center of the $m$ 'th class, which can be obtained by calculating the mean of the set of pixels belonging to the $m$ 'th class.

\section{Unsupervised Classification Method Based on AIS}

In the classification algorithm based on AIS, concepts such as antigen and antibody must be specific. Because each antibody should be one solution of the problem, it is defined as a vector whose length is equal to the number of classes, and each element of the vector records a coherency matrix of one class center. The antigens are defined as an array composed of all pixels. To estimate the similarity between antigens and antibodies, the Wishart distance is calculated as in Eq. (6). Table 1 shows the main terminologies and concepts in natural immune systems and in terms of PolSAR image classification.

The experimental procedure for classification can be described as follows:

1. Use the Lee refined filter with a $3 \times 3$ window to reduce speckle.

2. Decompose the coherency matrix of the PolSAR image to extract the entropy $H$ and the scattering angle $\alpha$.

3. Classify the pixels into $k$ classes according to the $H / \alpha$ plane ( $k=8$ in this study, compared with the $H / \alpha$-Wishart classifier).

4. Clone and mutate the antigens. The mutation operation will change the class of a pixel obeying a low probability. Because random mutation is prone to useless exploration, the possible choices are given as the neighbors of the pixel in the $H / \alpha$ plane. This means that if a pixel belongs to $Z_{9}$ in Fig. 1 , its mutated class can only range among $\left\{Z_{5}, Z_{6}, Z_{8}, Z_{9}\right\}$. The size of the antigen group is designated by $n$, and $n=80$ in this study.

5. Obtain the initial antibodies according to every antigen in the group. Find the best antibody by calculating the sum of the Wishart distances between each pixel and its corresponding class center.

6. Clone and mutate the best antibody to generate a group of size designated by $p$ ( $p=30$ in this study). The mutation operation is carried out according to the CSA and obeys Eq. (7).

$$
T_{c}=T_{c}-\lambda\left(T_{c}-T_{x}\right),
$$

where $T_{c}$ is the coherency matrix of a class center, $T_{x}$ is the coherency matrix of a random pixel belonging to this class, and $\lambda$ is the parameter modulating the mutation rate ( $\lambda$ was set to 0.07 in this study as an appropriate value according to numerous tests). 
Jie et al.: Polarimetric synthetic aperture radar image unsupervised classification method...

7. Find the best antibody in the group and inhibit the others.

8. Classify the pixels into $k$ classes according to the best antibody.

9. Judge whether the end condition has been met. If not, return to Step 6; otherwise exit the iteration and output the classification result.

\section{Experimental Results and Analysis}

Three sets of PolSAR images have been investigated to test the AIS-based algorithm in this study. The first consists of full-polarimetric SAR data for San Francisco Bay, California, obtained from NASA-JPL AIRSAR in 1992. The size of the experimental data set is $899 \times 651$ pixels. The region includes urban areas, ocean, vegetation, the Golden Gate Bridge, and other targets. The second consists of L-band PolSAR data for the Flevoland region from the NASA/JPL Laboratory (Airborne) AIRSAR sensor in 1989, with an azimuth resolution of $12.10 \mathrm{~m}$ and a distance resolution of $6.6 \mathrm{~m}$. The feature types in the experimental area are relatively simple; most are croplands of rectangular shape, including grassland, potatoes, alfalfa, wheat, soybeans, sugar beets, peas, and other target surface features. The size of the experimental data set is $293 \times 433$ pixels. The third consists of X-band full-polarimetric high-resolution SAR data for Lingshui Town in Hainan Province in 2010. The original size of this data set is $5001 \times 7893$ pixels. The region includes airport runways, urban areas, pools, and various kinds of croplands, such as red peppers, betel palms, mangoes, papayas, and rice paddies. Because the original image is too large for analysis, two subareas were selected for the experiments.

The first experiments were performed with the first data set and with the AIS algorithm mutation probability set to 0.05 . And the Fig. 2 shows the Pauli RGB image of the experiment area. Compared Fig. 3 with Fig. 4, it is clear that the classification of the ocean part with the CSA is better than with $H / \alpha$-Wishart classification method because the ocean contains only lowentropy and medium-entropy surface scattering, with the majority being low-entropy surface scattering.

Although the $H / \alpha$-Wishart classification method can characterize the scattering mechanism of surface features ${ }^{20,21}$ its classification of different surface features using the same scattering mechanism is not precise enough because there is not a one-to-one relationship between surface

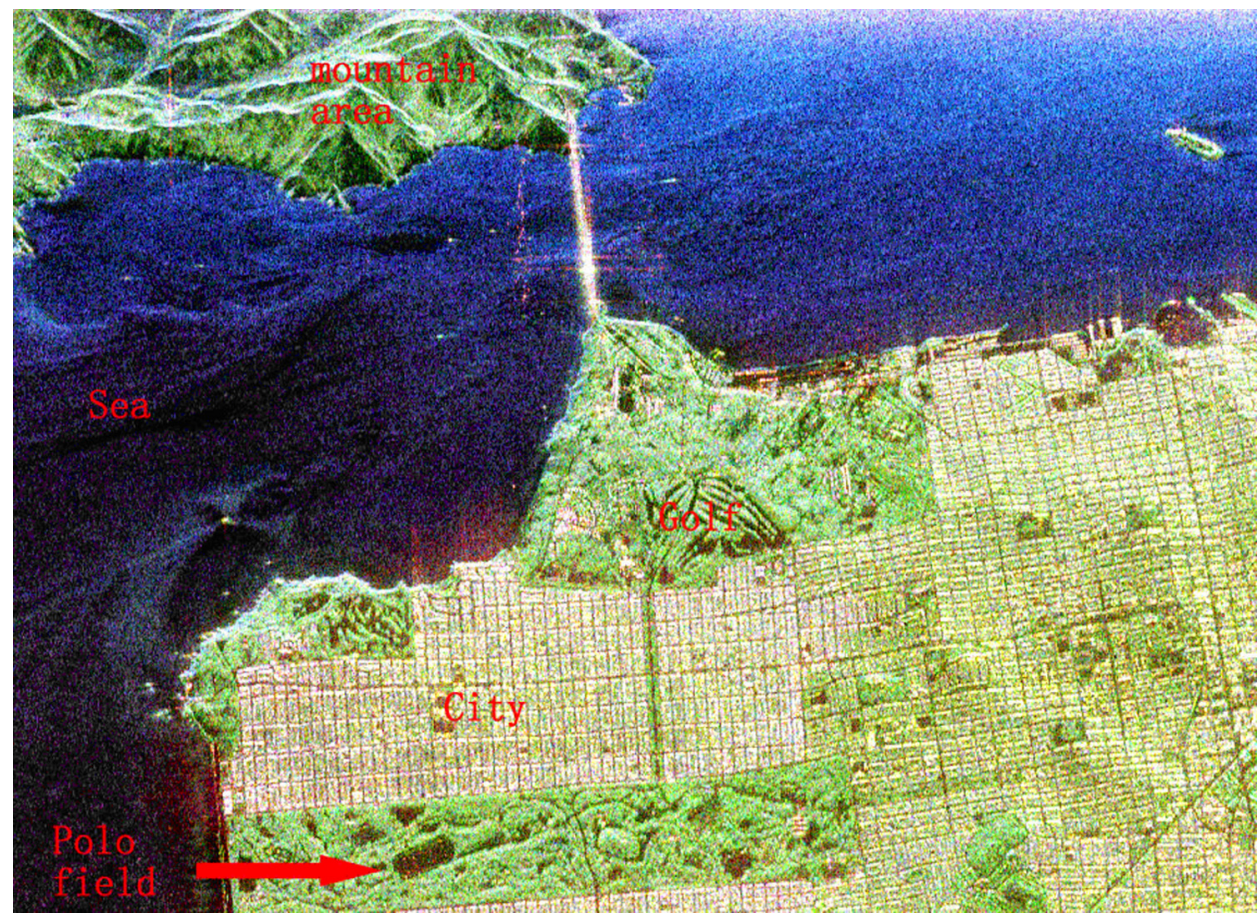

Fig. 2 Pauli RGB experimental composite image of the San Francisco Bay area. 


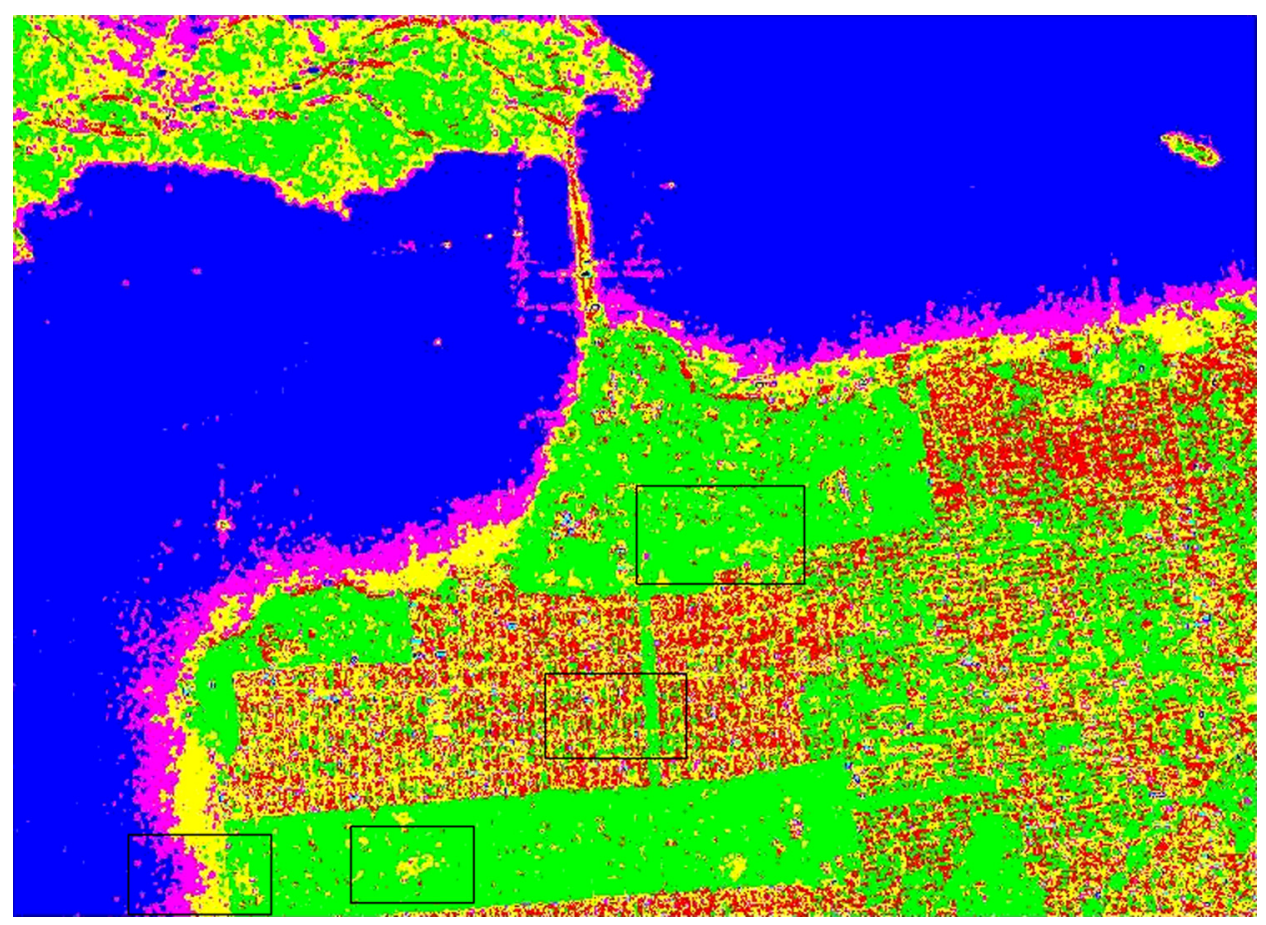

Fig. $3 H / \alpha$-Wishart classification result.

features and scattering mechanism. Both vegetation and urban areas contain both mediumentropy and multiple scattering components and, therefore, they were confused in the image results.

As shown by the $H / \alpha$-Wishart classification results in Fig. 5(b), the same type of surface feature with a variety of scattering mechanisms may be classified falsely into multiple categories. For instance, vegetation in the golf course in Fig. 5(b) with even and multiple scattering is seriously misclassified. Overall, the result shows a serious lack of texture information, and outlines

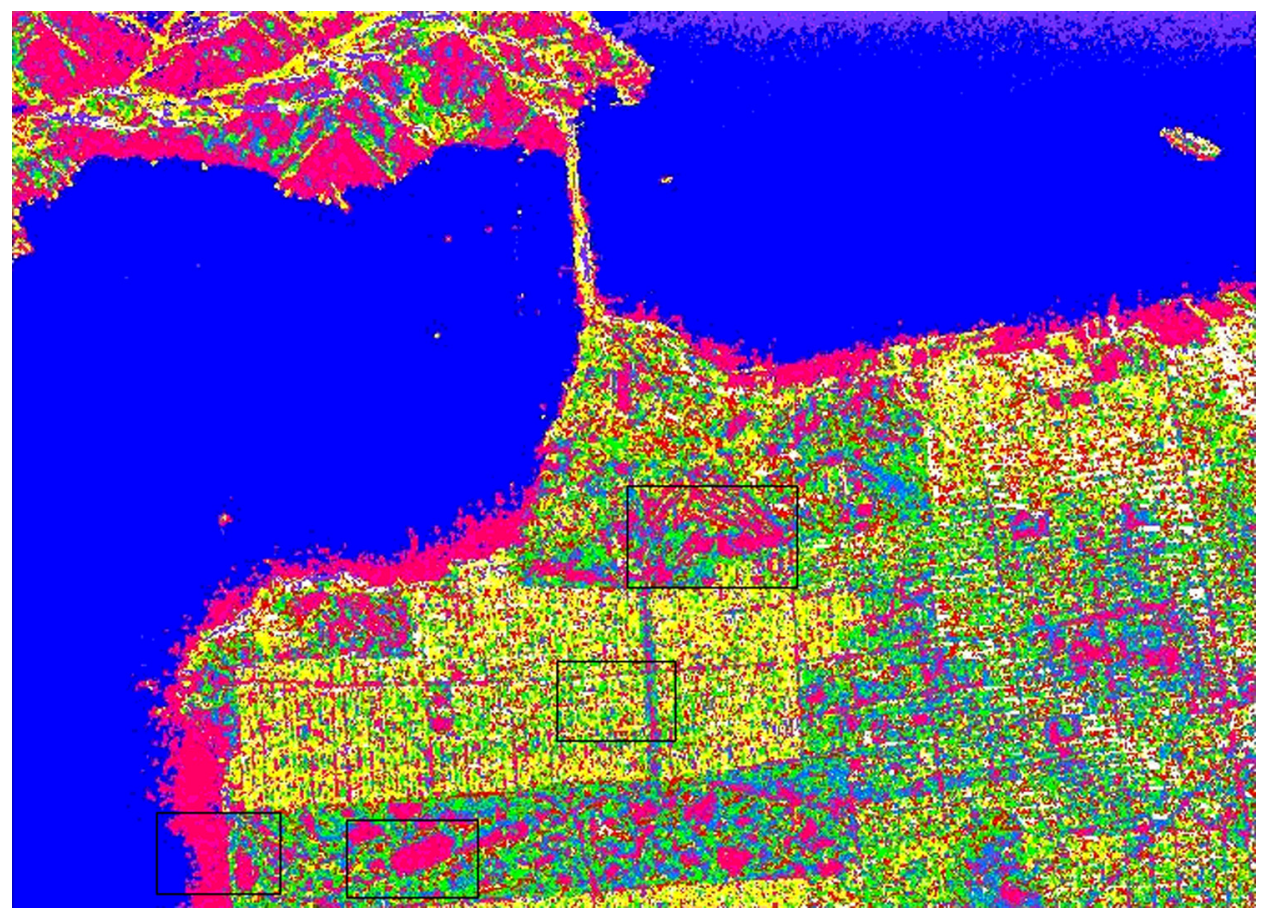

Fig. 4 Classification result of the clonal selection algorithm. 
Table 1 Comparison of concepts and terminologies in natural immune systems (NIS) and in an artificial immune system (AIS) for PolSAR image classification.

\begin{tabular}{lll}
\hline \hline IS concepts & \multicolumn{1}{c}{ NIS } & \multicolumn{1}{c}{ AIS } \\
\hline Antigens & $\begin{array}{l}\text { Harmful invasive cells, such as } \\
\text { bacteria and viruses. }\end{array}$ & $\begin{array}{l}\text { An array composed of the coherency } \\
\text { matrix of all pixels. }\end{array}$ \\
Antibodies & Immune B cells. & $\begin{array}{l}\text { Vector composed of the coherency } \\
\text { matrix of every class center. }\end{array}$ \\
Purpose of IS & $\begin{array}{l}\text { Generate appropriate immune } \\
\text { cells to recognize and kill antigens. }\end{array}$ & $\begin{array}{l}\text { Finding a group of class centers that can } \\
\text { minimum the sum of the distance of each } \\
\text { pixel to its corresponding class center. }\end{array}$ \\
$\begin{array}{ll}\text { Antigen } \\
\text { recognition }\end{array}$ & $\begin{array}{l}\text { Binding the surface of immune cells } \\
\text { with antigens. The best-matching } \\
\text { immune cells recognize the invasive } \\
\text { cell. }\end{array}$ & $\begin{array}{l}\text { By calculating the Wishart distance between } \\
\text { the coherency matrix of a pixel and the class } \\
\text { center of the class to which the pixel belongs. }\end{array}$ \\
\hline \hline
\end{tabular}

are not clear. By contrast, when using the CSA illustrated in Fig. 5(a), the classification results are greatly improved. A variety of surface features have been distinguished, the texture of vegetation appears clearer, sea levels are more apparent, and the classification of vegetation is better.

From comparison of the four extracted parts, it can be seen that the polo field in Fig. 5(b) is not obvious, while the polo field in Fig. 5(a) is an oval-shaped region with a smooth outline. Most of the beach A is identified by medium-entropy surface scattering, which corresponds to the actual situation. The golf course in Fig. 5(a) is classified clearly, while the golf course in Fig. 5(b) is almost impossible to identify. Although vegetation and urban areas are seriously confused in Fig. 5(b), the texture of urban areas and roads in Fig. 5(b) is quite clear. Overall, the classification results of the CSA are better.

To verify further the validity of the classification algorithm, the second set of PolSAR data was used for another set of experiments, and a quantitative analysis was performed using a confusion matrix. Figure 6 shows an RGB composite image of the region. The red, green, and blue components of the composite image were obtained using the three parameters $|\mathrm{HH}-\mathrm{VV}|,|\mathrm{HV}|$, and $|\mathrm{HH}+\mathrm{VV}|$ derived from the Pauli decomposition. The mutation probability in the AIS algorithm was set to 0.05 .

From visual observation of Fig. 7, the classification result of the image is quite smooth. Some areas were not distinguished, such as peas and sugar beets, while other types were misclassified, such as potatoes. However, the majority of the surface features, such as peas and sugar beets, can be identified in the CSA classification results shown in Fig. 8. Potatoes were also classified correctly, and the improvement is clear.

To evaluate the classification accuracy of the CSA, Fig. 6(b) shows a reference image of the real surface features. Test samples from the image were selected randomly (Fig. 9), and confusion matrices were obtained for the $H / \alpha$-Wishart and CSA classifications.
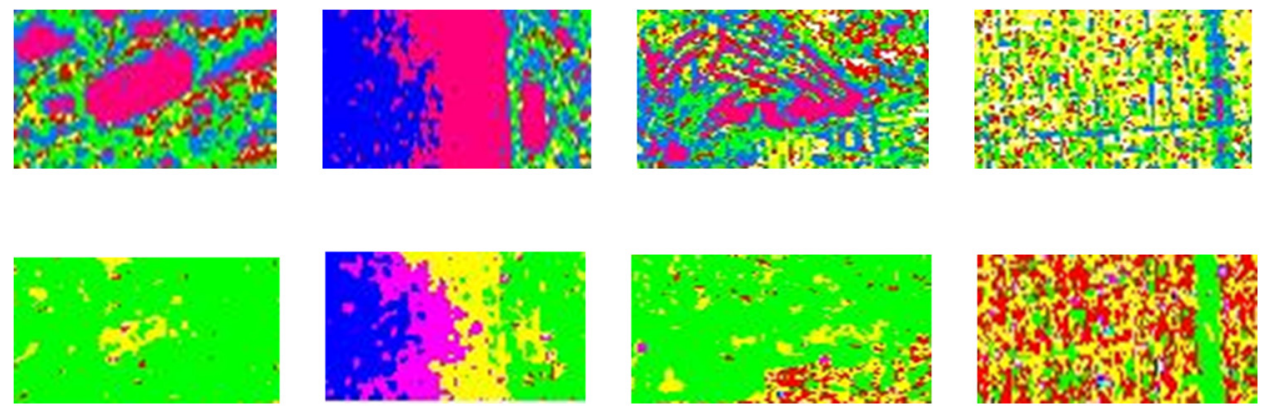

Fig. 5 Comparison of classification results. 
Jie et al.: Polarimetric synthetic aperture radar image unsupervised classification method...
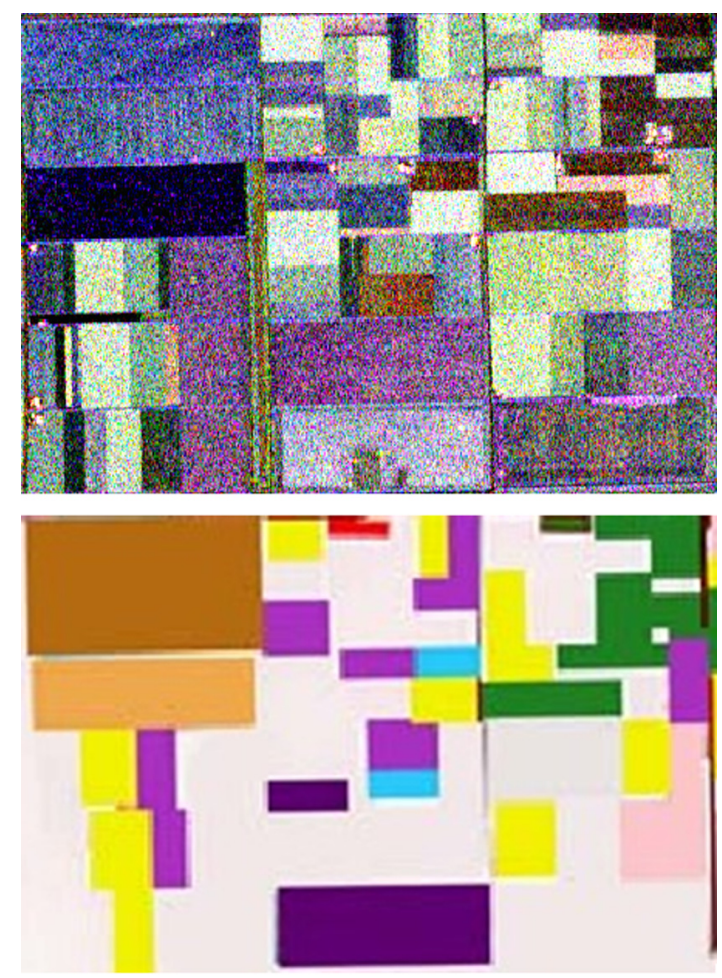

Fig. 6 The Flevoland experimental data. (a) Pauli RGB image. (b) Real surface features.

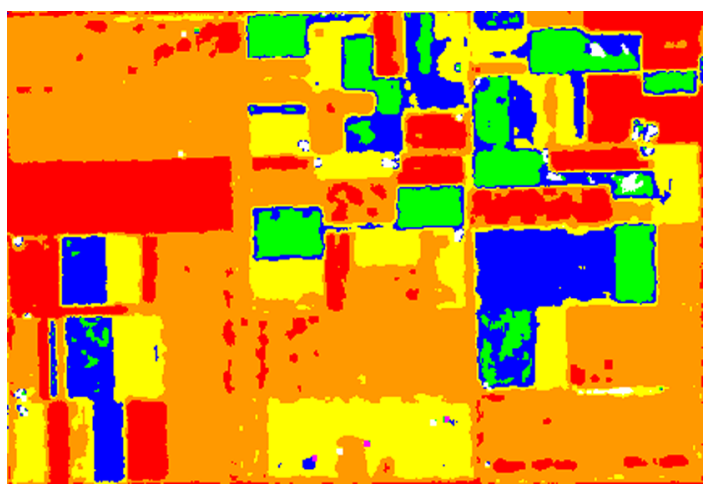

Fig. $7 H / \alpha$-Wishart classification result.

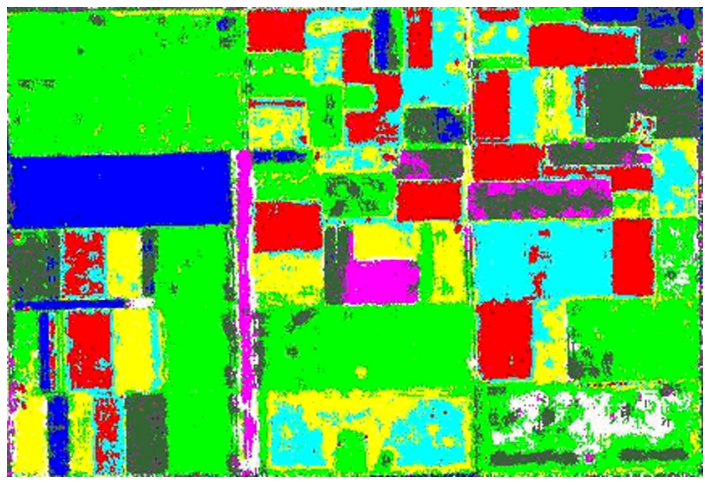

Fig. 8 Clonal selection algorithm classification result. 
Jie et al.: Polarimetric synthetic aperture radar image unsupervised classification method...

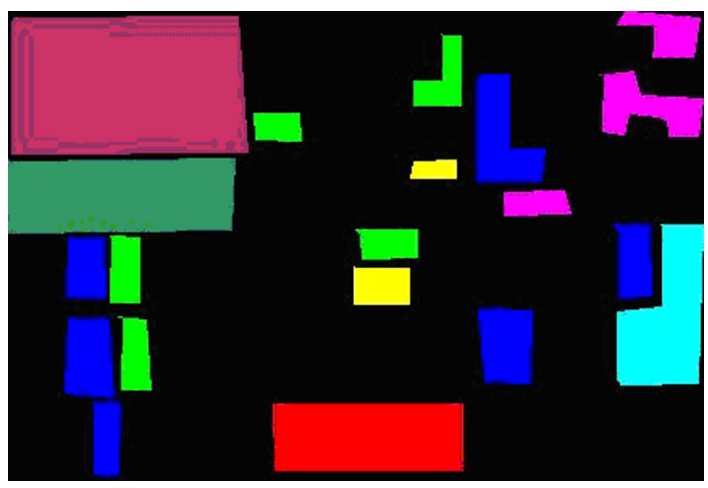

Fig. 9 Regions of interest for the Flevoland image.

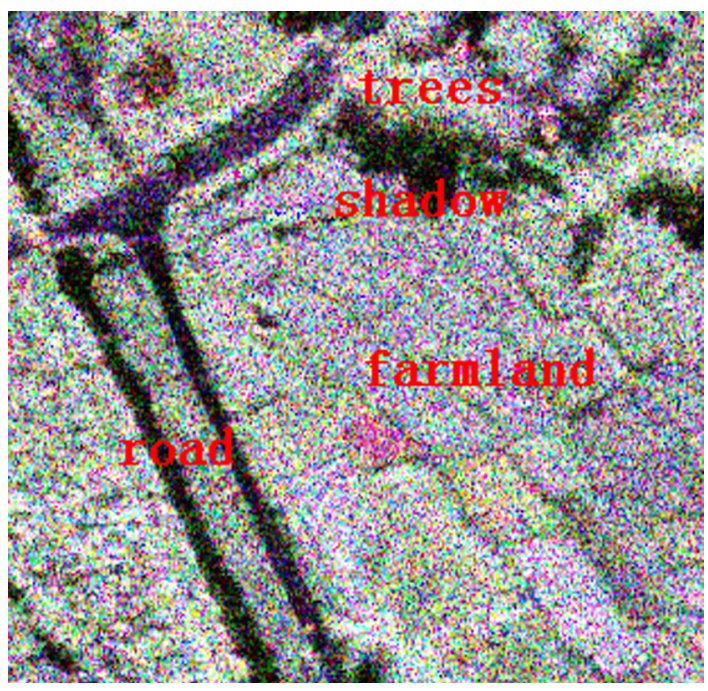

Fig. 10 Pauli RGB experimental composite image of the Lingshui area (subset one).
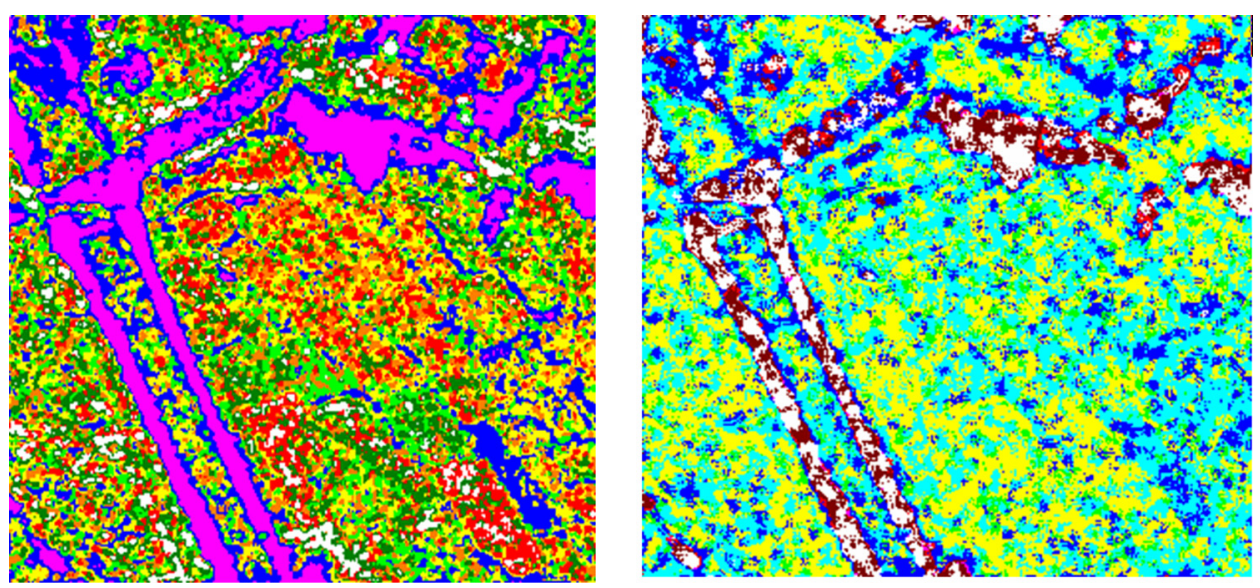

Fig. 11 Classification result. (a) $H / \alpha$-Wishart. (b) Clonal selection algorithm.

As can be seen from Table 2, the accuracy of the CSA is greater than that of the $H / \alpha$-Wishart method, both in terms of overall accuracy and the kappa coefficient. For some categories, such as rape and bare soil, the mapping accuracy and precision of the clonal selection method were $>90 \%$. The $H / \alpha$-Wishart classification algorithm distinguished only four vegetation categories, misclassified peas and beets into the same category, divided potatoes into two categories, and made no distinction between grassland, rape, and other surface features, resulting in lower classification accuracy. The CSA achieved a better classification result. 
Jie et al.: Polarimetric synthetic aperture radar image unsupervised classification method...

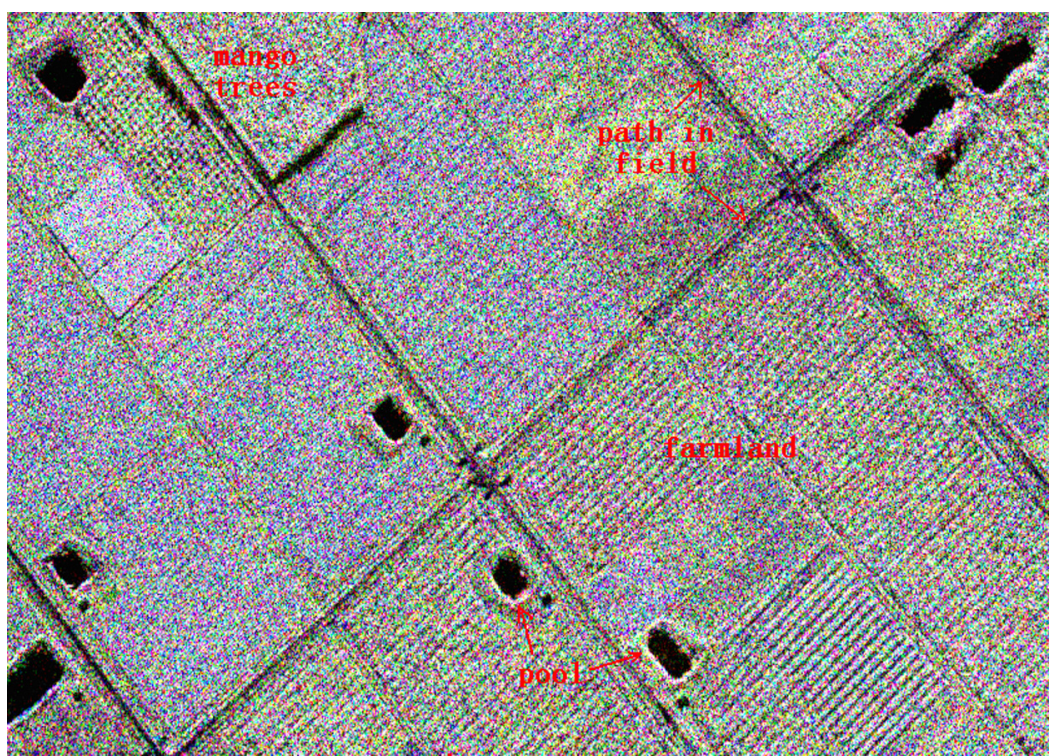

Fig. 12 Pauli RGB experimental composite image in the Lingshui area (subset two).

To verify that the CSA has stable performance, a set of experiments was performed using the third data set. The experiment image and the results are showed in Figs. 10 and 11.

Because the experimental data are of high resolution in the farmland area, neither of the two algorithms exhibited good performance. The $H / \alpha$-Wishart classification result combined the road and the shadow of the trees into a single category. In the CSA result, although these two features could still not be separated distinctly, a further classification could be performed

Table 2 Clonal selection algorithm and $H / \alpha$-Wishart algorithm confusion matrices.

\begin{tabular}{|c|c|c|c|c|c|c|c|}
\hline & Plot & $\begin{array}{c}\text { User } \\
\text { accuracy (\%) }\end{array}$ & $\begin{array}{c}\text { Mapping } \\
\text { accuracy (\%) }\end{array}$ & $\begin{array}{l}\text { Misclassification } \\
\text { error (\%) }\end{array}$ & $\begin{array}{l}\text { Leakage } \\
\text { point error } \\
(\%)\end{array}$ & $\begin{array}{l}\text { Overall } \\
\text { accuracy } \\
(\%)\end{array}$ & $\begin{array}{c}\text { Kappa } \\
\text { coefficient }\end{array}$ \\
\hline \multirow{7}{*}{$\begin{array}{l}H / \alpha \text {-Wish } \\
\text { art }\end{array}$} & $\begin{array}{l}\text { Bare soil } \\
\text { Beets }\end{array}$ & $\begin{array}{l}92.32 \\
48.16\end{array}$ & $\begin{array}{l}90.02 \\
92.67\end{array}$ & $\begin{array}{c}7.68 \\
51.84\end{array}$ & $\begin{array}{l}9.98 \\
7.33\end{array}$ & \multirow{7}{*}{72.51} & \multirow{7}{*}{0.5627} \\
\hline & Potatoes & 84.33 & 94.55 & 15.67 & 5.45 & & \\
\hline & Wheat & 60.35 & 98.47 & 39.65 & 1.53 & & \\
\hline & Lawn & 0.00 & 0.00 & 0.00 & 100 & & \\
\hline & Pea & 0.00 & 0.00 & 100 & 100 & & \\
\hline & Rape & 0.00 & 0.00 & 0.00 & 100 & & \\
\hline & Alfalfa & 7.76 & 0.49 & 92.24 & 99.51 & & \\
\hline \multirow{6}{*}{$\begin{array}{l}\text { Clonal } \\
\text { selection } \\
\text { algorithm }\end{array}$} & $\begin{array}{l}\text { Bare soil } \\
\text { Beets }\end{array}$ & $\begin{array}{l}97.26 \\
62.28\end{array}$ & $\begin{array}{l}94.79 \\
85.54\end{array}$ & $\begin{array}{c}2.74 \\
37.72\end{array}$ & $\begin{array}{c}5.21 \\
14.46\end{array}$ & \multirow{7}{*}{80.74} & \multirow{7}{*}{0.7297} \\
\hline & Potatoes & 95.50 & 65.39 & 4.50 & 34.61 & & \\
\hline & Wheat & 57.51 & 39.54 & 42.49 & 60.46 & & \\
\hline & Lawn & 56.61 & 70.76 & 43.39 & 29.24 & & \\
\hline & Pea & 60.55 & 55.59 & 39.45 & 44.41 & & \\
\hline & Rape & 92.76 & 88.50 & 7.24 & 11.50 & & \\
\hline Alfalfa & 20.57 & 70.66 & 79.43 & 29.34 & & & \\
\hline
\end{tabular}




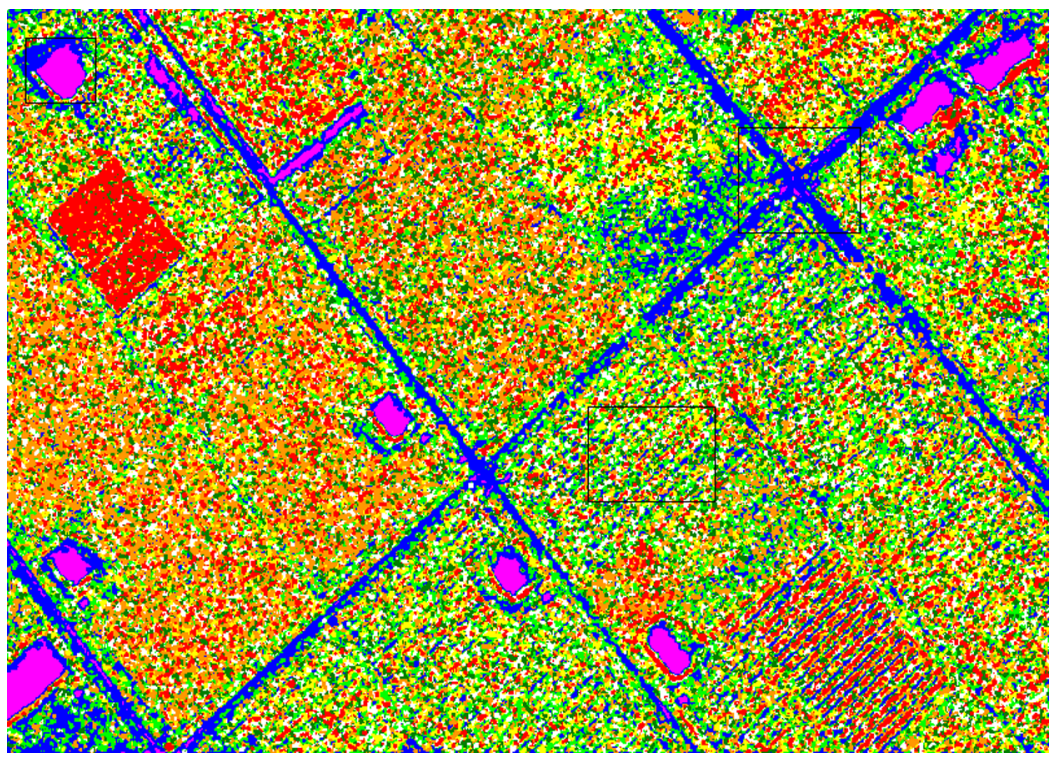

Fig. $13 H / \alpha$-Wishart classification result.

in this area. Because the pixels representing roads were further divided into two categories (brown and white) in the results of the AIS algorithm, it is apparent that the AIS algorithm revealed additional information in homogeneous regions and can explore polarimetric information to a greater extent.

The first and second experiments have already proved that the CSA can improve the quality of classification results for classic PolSAR data. And the forth experiment are applied with the second subset of Lingshui image which contains mango trees, farmland, path in field and pools (showed in Fig. 12). Figure 15, which is extracted from Figs. 13 and 14, illustrates the improvement achieved using the CSA. For the pixels representing the pool in Figs. 15(a) and 15(b), the two algorithms achieve similar performance, but around the pool in Fig. 15(b), the bare soil has been classified into two categories in a reasonable way. This phenomenon can also be seen with the country road in Fig. 15(b). For the papaya field, the result in Fig. 15(a) shows more randomness and mass, but in Fig. 15(b), more texture information has been retrieved.

From the above discussion, it can be concluded that the CSA can achieve better classification results and exhibits stable performance on various kinds of data. This difference occurs because

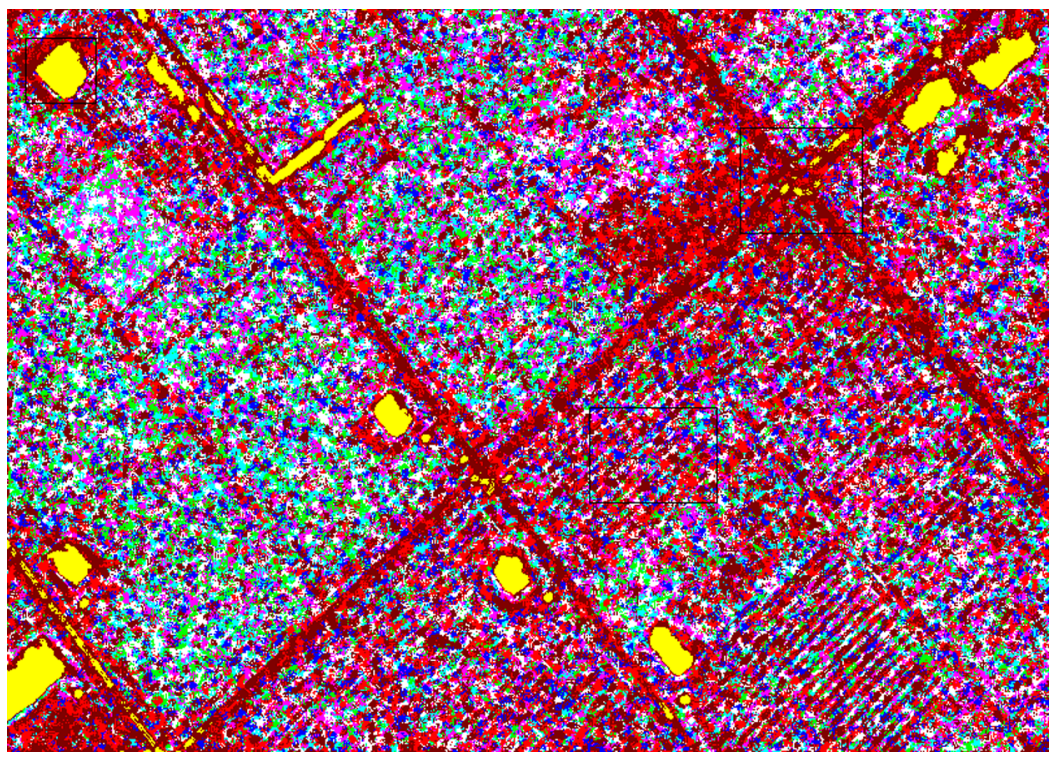

Fig. 14 Clonal selection algorithm classification result. 

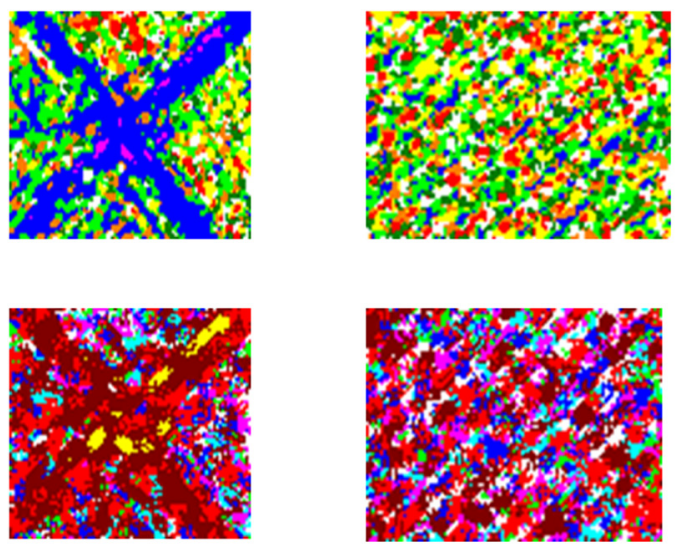

Fig. 15 Detailed comparison of classification results. (a) Country road, papaya field, pool. (b) Country road, papaya field, pool.

the $H / \alpha$-Wishart method can search only cluster centers according to a rigid rule. The classification results of the $H / \alpha$-Wishart algorithm are greatly affected by the initial classification, but the AIS is a kind of search strategy that is capable of global optimization.

\section{Conclusions}

To increase classification precision, a new method has been proposed for PolSAR classification based on the CSA. By treating pixels as antigens and class center groups as antibodies, the novel algorithm takes advantage of global search and self-learning to obtain the global optimal solution quickly in clustering problems. Experimental results show that the proposed algorithm with an improved initialization method and a novel mutation operation is better than the $H / \alpha$-Wishart classification and is a more effective PolSAR classification algorithm than its competitors, with stable performance.

\section{Acknowledgments}

This study is supported by two Chinese Foundation: 863 Key Project "Object Oriented Features of High Confidence SAR Interpretation System" and Fourth Project "Object Oriented SAR Images High Credible Interpretation Technique" (No. 2011AA120404) and Special Foundation of Geographical Condition Monitoring (No. B1288).

\section{References}

1. J. S. Lee et al., "Unsupervised classification using polarimetric decomposition and the complex Wishart classifier," IEEE Trans. Geosci. Remote Sens. 37(5), 2244-2266 (1999), http:// dx.doi.org/10.1109/36.789621.

2. P. Lombardo et al., "Optimum model-based segmentation techniques for multifrequency polarimetric SAR images of urban areas," IEEE Trans. Geosci. Remote Sens. 41(9), 1959-1975 (2003), http://dx.doi.org/10.1109/TGRS.2003.814632.

3. S. R. Cloude and E. Pottier, "An entropy based classification scheme for land applications of polarimetric SAR," IEEE Trans. Geosci. Remote Sens. 35(1), 68-78 (1997), http://dx.doi .org/10.1109/36.551935.

4. S. Ning, "Artificial immune optimization algorithm and applications," MS Thesis, Harbin Institute of Technology, Harbin (2006).

5. Y. Zhong et al., "A supervised artificial immune classifier for remote-sensing imagery," IEEE Trans. Geosci. Remote Sens. 45(12), 3957-3966 (2007), http://dx.doi.org/10.1109/ TGRS.2007.907739. 
Jie et al.: Polarimetric synthetic aperture radar image unsupervised classification method...

6. Y. Yamaguchi et al., "Classification of terrain by implementing the correlation coefficient in the circular polarization basis using X-band POLSAR data," IEICE Trans. Commun. E91.B(1), 297-301 (2008), http://dx.doi.org/10.1093/ietcom/e91-b.1.297.

7. E. Pottier and J. S. Lee, "Application of the H/A/alpha polarimetric decomposition theorem for unsupervised classification of fully polarimetric SAR data based on the Wishart distribution," in Proc. of Committee on Earth Observing Satellites SAR Workshop, pp. 335-340, European Space Agency, Paris (2000).

8. K. S. Chen et al., "Classification of multifrequency polarimetric SAR image using a dynamic learning neural network," IEEE Trans. Geosci. Remote Sens. 34(3), 814-820 (1996), http://dx.doi.org/10.1109/36.499786.

9. J. H. Carter, "The immune system as a model for pattern recognition and classifications," $J$. Am. Med. Inform. Assoc. 7(1), 28-41 (2000), http://dx.doi.org/10.1136/jamia.2000.0070028.

10. S. Kiranyaz et al., "Collective network of binary classifier framework for polarimetric SAR image classification: an evolutionary approach," IEEE Trans. Geosci. Remote Sens. 42(4), 1169-1185 (2012), http://dx.doi.org/10.1109/TSMCB.2012.2187891.

11. J. Timmis and M. Neal, "An artificial immune system for data analysis," Biosystems 55(1-3), 143-150 (2000), http://dx.doi.org/10.1016/S0303-2647(99)00092-1.

12. Y. Zhong et al., "An unsupervised artificial immune classifier for multi/hyperspectral remote sensing imagery," IEEE Trans. Geosci. Remote Sens. 44(2), 420-431 (2006), http://dx.doi .org/10.1109/TGRS.2005.861548.

13. J. Licheng and D. Haifeng, The Computation Study and Distinguish of Immunity Optimization, The Science, Beijing (2006).

14. Q. Xiao-Ning, "Research and application of classification method based on artificial immune system," MS Thesis, Hunan University, Changsha (2008).

15. D. Dasgupta, Ed., Artificial Immune Systems and Their Application, Springer-Verlag, Berlin, Heidelberg (1999).

16. J. J. VanZyl, H. A. Zebker, and C. Elachi, "Imaging radar polarization signatures: theory and observation," Radio Sci. 22(4), 529-534 (1987), http://dx.doi.org/10.1029/ RS022i004p00529.

17. W. Chao and H. Zhang, The Image Procession of Polarimetric Synthetic Aperture Radar, The Science, Beijing (2008).

18. S. R. Cloude and K. P. Papathanassiou, "Polarimetric SAR interferometry," IEEE Trans. Geosci. Remote Sens. 36(5), 1551-1565 (1998), http://dx.doi.org/10.1109/36.718859.

19. P. Yu, A. K. Qin, and D. A. Clausi, "Unsupervised polarimetric SAR image segmentation and classification using region growing with edge penalty," IEEE Trans. Geosci. Remote Sens. 50(4), 1302-1317 (2012), http://dx.doi.org/10.1109/TGRS.2011.2164085.

20. J. S. Lee and M. R. Grunes, "Classification of multi-look polarimetric SAR imagery based on complex Wishart distribution," Int. J. Remote Sens. 15(11), 2299-2311 (1994), http://dx .doi.org/10.1080/01431169408954244.

21. J. S. Lee, M. R. Grunes, and E. Pottier, "Quantitative comparison of classification capability: fully polarimetric versus dual and single-polarization SAR," IEEE Trans. Geosci. Remote Sens. 39(11), 2343-2351 (2001), http://dx.doi.org/10.1109/36.964970.

Yu Jie is a professor in the College of Resource Environment and Tourism and the State Key Laboratory Incubation Base of Urban Environmental Processes and Digital Simulation at Capital Normal University. She is engaged in teaching and research about geographic information systems (GIS) and remote sensing. Her main research interests are in remotely sensed imagery processing and application of GIS.

Wang Gang received his MS degree in information engineering from Wuhan University, China, in 2011. Now he works in remote-sensing image processing at the Institute of Surveying and Mapping, Qingdao.

Zhu Teng received his BS degree in soft engineering from Wuhan University, China, in 2010. And now he is studying remote-sensing image processing, artificial intelligence, and pattern recognition for the $\mathrm{PhD}$ in the School of Remote Sensing and Information Engineering, Wuhan University, China. 
Jie et al.: Polarimetric synthetic aperture radar image unsupervised classification method...

Li Xiaojuan is a professor in the College of Resource Environment and Tourism and the Beijing Key Laboratory of Resource Environment and Geographic Information System at Capital Normal University. She is engaged in teaching and research about resource and environment information systems and environmental remote sensing.

Yan Qin is the vice president in the China Academy of Surveying and Mapping and also a professor in the Capital Normal University. She is engaged in the research on photogrammetry, remote sensing, resource and environment monitoring. 